\title{
Unifying the Early-Time Inflationary Era with Late- Time Dark Epoch Universe: The Case of Modified Gravity $^{+}$
}

\author{
Sergei Odintsov ${ }^{1,2}$ \\ 1 Space Research Inst. (CSIC-IEEC), Cr.can Magrans s/n, 08093 Barcelona, Spain; odintsov@ieec.uab.es \\ 2 ICREA, 08093 Barcelona, Spain \\ + Presented at Symmetry 2017-The First International Conference on Symmetry, Barcelona, Spain, \\ 16-18 October 2017.
}

Published: 4 January 2018

\begin{abstract}
We discuss various theories of modified gravity which aim to describe the whole evolution of the universe, from early-time inflation via radiation/matter dominance and finally to the dark energy era. Such unified evolution may be described in frames of the same modified gravity. Special attention is paid to $F(R)$ gravity where $R$ is scalar curvature and modified GaussBonnet gravity. The modified gravity solves the problem of universe acceleration without the need to introduce inflation or unknown dark fluid. The explicit models of such $F(R)$ gravity are presented where quantum gravity effects may also be taken into account. The possible generalizations of modified gravity to non-local gravity, string-inspired gravity, and teleparallel gravity are briefly discussed. A number of applications such as the qualitative change of mass-radius relation in neutron stars or the anti-evaporation effect in multiple-horizon black holes are mentioned.
\end{abstract}

Keywords: gravity; cosmology

(C) 2018 by the author. Licensee MDPI, Basel, Switzerland. This article is an open access article distributed under the terms and conditions of the Creative Commons Attribution (CC BY) license (http://creativecommons.org/licenses/by/4.0/). 